\title{
An Initial Value Calibration Method for the Wheel Force Transducer Based on Memetic Optimization Framework
}

\author{
Guoyu Lin, ${ }^{1}$ Weigong Zhang, ${ }^{1}$ Fan Yang, ${ }^{1}$ Han Pang, ${ }^{2}$ and Dong Wang ${ }^{1}$ \\ ${ }^{1}$ School of Instrument Science and Engineering, Southeast University, China \\ ${ }^{2}$ School of Mechanical and Electrical Engineering, China University of Petroleum, China \\ Correspondence should be addressed to Guoyu Lin; andrew.lin@seu.edu.cn
}

Received 21 August 2013; Revised 2 October 2013; Accepted 10 October 2013

Academic Editor: Gelan Yang

Copyright (c) 2013 Guoyu Lin et al. This is an open access article distributed under the Creative Commons Attribution License, which permits unrestricted use, distribution, and reproduction in any medium, provided the original work is properly cited.

\begin{abstract}
Some initial values of the wheel force transducer (WFT) change after being mounted in the vehicle. The traditional static calibration is inadequate to fully obtain these initial values. Aiming to this problem, an online initial value calibration method is proposed. The method does not require any additional calibration equipment or manual operation and just requires the vehicle mounted with the WFT to be driven on a flat road with constant speed. In this way, an initial value mode is constructed and then converted to an optimization problem. To solve this problem and acquire the right initial value, an improved Memetic framework based on particle swarm optimization (PSO) and Levenberg-Marquardt (LM) is adopted. To verify the effect of the proposed method, the real WFT data is used and the comparative test is carried out. The experiment result shows that the proposed method is superior to the traditional one and can improve the measurement accuracy effectively.
\end{abstract}

\section{Introduction}

When the vehicle is moving on the road, three-dimensional forces and three-dimensional torques are applied to the wheel, which is longitudinal force $F_{x}$, lateral force $F_{y}$, vertical force $F_{z}$, heeling moment $M_{x}$, twist torque $M_{y}$, and aligning torque $M_{z}$, respectively. The schematic diagram of wheel force is shown in Figure 1, where $O_{w} X_{w} Y_{w} Z_{w}$ is the wheel coordinate and its origin is the wheel center. The interaction between the vehicle and ground can be represented by the wheel force; therefore, sensing the forces/torque of wheel dynamically has been an important component of vehicle for the research of body vibration, suspension, and wheel dynamics as well as the performance matching of vehicle transmission and braking system [1-3].

Wheel force transducer (WFT) is a tool which can measure the wheel force effectively. For the increasing importance of wheel force, some world famous manufacturers in the automobile test field, including MTS Corporation, Michigan, Scientific Corporation, and Kistler Corporation, and so forth, have been devoted to the research of the WFT and have developed some WFT products. These WFT products have been used in many vehicle test fields and acquired acceptable performance. However, these WFT products are expensive and their detail technology information are not publicly available due to commercial reasons. These factors not only impede the popularization and application of the WFT, but also slow down the further development of the WFT. Southeast University has been devoted to research of the WFT for over ten years and developed some prototype, as shown in Figure 2, and published some papers in the field of static calibration, decoupling across dimension, and structure analysis [4-6]. However, there are still some inadequacies that need to be improved.

Essentially, the WFT is a multiple dimensional force sensor. The calibration is very important for the WFT because it is used to acquire the mathematical model between input and output of the sensor and affects the measurement accuracy directly. Thus, many scholars have focused on the research of calibration of multiple dimensional force sensor and proposed that a calibration platform is a good device for sensor calibration [7-10]. However, for the WFT, there are three initial values to be solved which are $\alpha_{0}, F_{\mathrm{CG} 0}, F_{\mathrm{AE} 0}$, where $\alpha_{0}$ is the initial installation angle of the WFT and $F_{\mathrm{CG} 0}$ and $F_{\mathrm{AE} 0}$ are the output of $B_{\mathrm{CG}}$ bridge and $B_{\mathrm{AE}}$ bridge of the WFT. Because of bolt force and wheel weight, these initial 


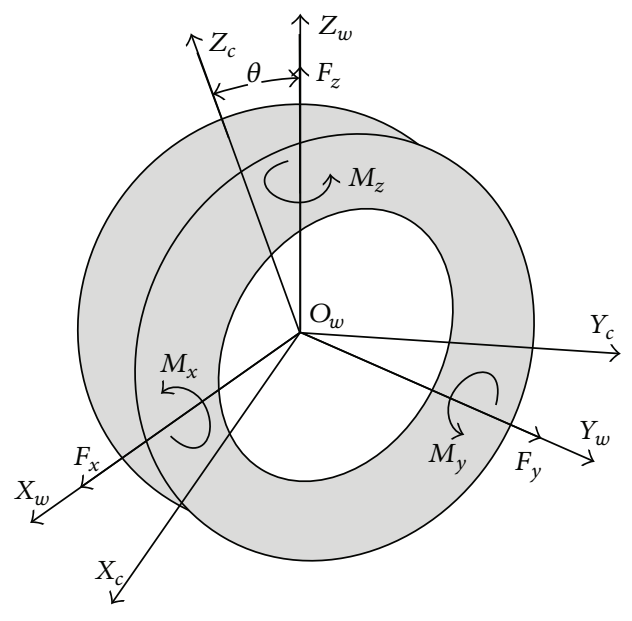

FIgURE 1: The diagram of six-dimensional forces of wheel.

values change after the sensor being bolted in the wheel and then installed in the vehicle. This is quite different from other multiple dimensional force sensors, like wrist force sensor and so on. Therefore, the classical static calibration method is inadequate to acquire the initial value of the WFT.

To handle the problem, the researchers proposed some methods. A traditional way to estimate the initial installation angle $\alpha_{0}$ of the WFT is shown in Figure 3, which is presented by Zhang [11]. If no longitudinal force $F_{x}$ is applied to the sensor, when the output of $B_{\mathrm{AE}}$ bridge reaches its maximum in a signal period, the rotating angle of the WFT can be considered as the initial angle $\alpha_{0}$. Based on it, Zhang proposes the scene can be simulated by pushing the vehicle to move slowly and manually. However, this method has two disadvantages: (1) pushing the vehicle to move manually is not suitable for large and heavy vehicles, (2) in the circumstance, the condition of non- $F_{x}$ is not established in real application. Therefore, by adopting the method, the error exists inevitable. Liu et al. proposed a weighing on-vehicle calibration method for the WFT as shown in Figure 4 [12]. The vehicle is lifted with a jack. Then the specific two beams of the WFT's elastic body are rotated to a horizontal position, successively, and the output of $B_{\mathrm{CG}}$ bridge and $B_{\mathrm{AE}}$ bridge are considered as $F_{\mathrm{CG} 0}$ and $F_{\mathrm{AE} 0}$. This method achieves good effect in real application; however judging the horizontal position manually will cause error and the whole process is tedious.

The two methods shown above actually belong to an online calibration method. Now, in multiple dimensional force sensor fields, some researchers have focused on the online calibration and proposed some practical methods. Wang proposed an autostatic calibration method where the force sensor that is exerted with a known mass weight is rotating, and then the force exerted on the force sensor by the weight is computed to acquire the calibration matrix [13]. Kourosh presents a novel approach for the calibration of the force/torque sensors of space robots based on the dynamic effects of the wrench (force and torque) applied on a payload with known mass properties [14]. Kim and Sun introduce an effective shape-from-motion method to calculate the calibration matrix which refers to the fact that the shape of the force sensor can be recovered by knowing the theoretical rank of the shape and applying arbitrary motion to the force $[15,16]$. Nevertheless, these online calibration methods are not applicable to the WFT, for example, a known mass weight cannot be exerted on a rolling wheel for safety reasons.

From the related work review, it is clear that the existing methods are time-consuming, and the solved initial value is not correct enough, which results in the inaccuracy of measurement. Therefore, in the paper, an online initial value calibration method is put forward. The rest of the paper is organized as follows. In Section 2, we introduce the WFT's structure used in the paper. In Section 3, the details of the proposed method are elaborated by introducing the initial value mode and showing how to exploit the Memetic algorithm to get the initial value of the WFT. To illustrate the efficiency of the proposed method, in Section 4 we analyze the experimental results on real sampled datasets. Finally, we draw conclusions in the last section of the paper and discuss future work on this topic.

\section{The Overall Structure of the Adopted WFT}

The WFT depicted in the paper is developed by Southeast University and can measure two forces and one torque which is longitudinal force $F_{x}$, lateral force $F_{y}$, and twist torque $M_{y}$. The overall structure of the exploited 3-axis WFT is shown in Figure 5(a). It is composed of the elastic body, reforming rims, intermediate flange, sample module, and transfer module. The wheel rims are reformed to connect with the elastic body by bolt, and the intermediate flange is used to protect bridge circuit in the elastic body from water and dirt. The sample module is used to sample the wheel force and rotation angle. Then it sends them to the transfer module by wireless module. It connects to the elastic body by intermediate flange and rotates with the rolling wheel. The transfer module connects to the sample module by the bearing and does not rotate with the rolling wheel. It can receive the data from the sample module by wireless module too, and it can forward the data to the data acquisition devices by CAN bus. The electrical flowchart is show in Figure 5(b).

Figure 6 depicts the mechanical structure of the 3-axis WFT's elastomer. According to the requirement of axial structure size of the wheel, the eight spoke structure is exploited. The elastic body consists of an inner ring, an outer ring, and eight elastic beams. The eight elastic beams are labeled as $\mathrm{A}-\mathrm{H}$ and distributed uniformly between the inner ring and the outer ring. $O_{c} X_{c} Y_{c} Z_{c}$ is the elastomer coordinate where the CG-beam coincides with $X_{c}$ axis and the AEbeams coincides with $Z_{c}$ axis. Resistance strain gauges $R 1-$ $R 16$ are placed in the elastic beam to sense its deformation caused by the wheel force. $R 5$ and $R 6$ in C-Beam and $R 13$ and $R 14$ in G-Beam are connected to form the $B_{\mathrm{CG}}$ bridge to measure the force applied along $Y_{c}$ axis. $R 1$ and $R 2$ in $\mathrm{A}-$ Beam and $R 9$ and $R 10$ in E-Beam are connected to form the $B_{\mathrm{AE}}$ bridge to measure the force applied along $Z_{c}$ axis. Other strain gauges in B-Beam, D-Beam, F-Beam, and $\mathrm{H}$-Beam are 

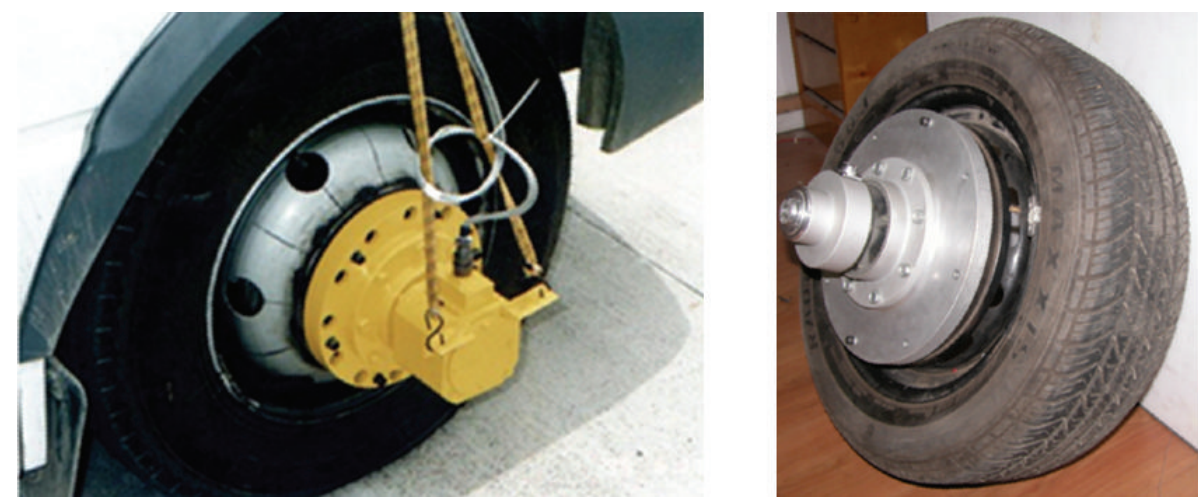

FIGURE 2: The WFT prototypes developed by Southeast University.

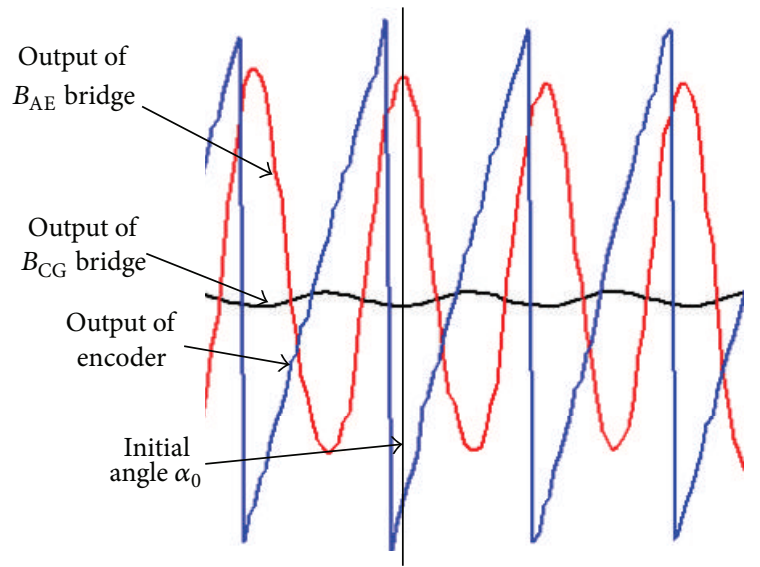

Figure 3: The outputs of the WFT.

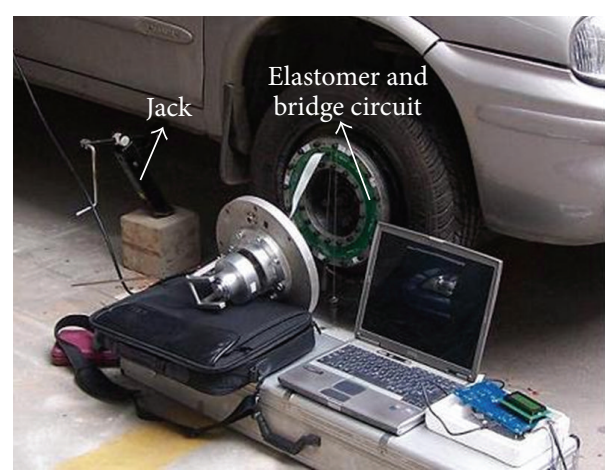

FIGURE 4: The weighing on-vehicle calibration method.

connected to form the $B_{\mathrm{BDFH}}$ bridge to measure the torque applied around $Y_{c}$ axis.

\section{Initial Value Calibration Method}

3.1. Initial Value Mode. As shown in Figure 6, $O_{c} X_{c} Y_{c} Z_{c}$ is the elastomer coordinate and it rotates with the wheel. The C-G beam is coincident with $X_{c}$ axis and the A-E beams is coincident with $Z_{c}$ axis. So the outputs of the three bridges are all represented in $O_{c} X_{c} Y_{c} Z_{c}$. However, the wheel force is represented in $O_{w} X_{w} Y_{w} Z_{w}$. The relationship between $O_{c} X_{c} Y_{c} Z_{c}$ and $O_{w} X_{w} Y_{w} Z_{w}$ is shown in Figure 1. When the wheel is rolling, $O_{c} X_{c} Y_{c} Z_{c}$ rotates around the $Y_{w}$ axis, and the rotation angle between $O_{c} X_{c} Y_{c} Z_{c}$ and $O_{w} X_{w} Y_{w} Z_{w}$ is denoted as $\theta$. Furthermore, the outputs of three bridges are sampled by $\mathrm{AD}$ converter, and the wheel force/torque is represented in unit $\mathrm{KN}$ or $\mathrm{KN} \cdot \mathrm{m}$. Therefore, according to the principle of the WFT, a transformation should be carried out to acquire the wheel force from the outputs of three bridges, which is shown below. Consider

$$
\left[\begin{array}{c}
F_{x} \\
F_{z} \\
M_{y}
\end{array}\right]=\left[\begin{array}{ccc}
\cos \theta & \sin \theta & 0 \\
-\sin \theta & \cos \theta & 0 \\
0 & 0 & 1
\end{array}\right]\left[\begin{array}{c}
f^{1}\left(v F_{\mathrm{CG}}\right)-F_{\mathrm{CG} 0} \\
f^{2}\left(v F_{\mathrm{AE}}\right)-F_{\mathrm{AE} 0} \\
f^{3}\left(v F_{\mathrm{BDFH}}\right)-F_{\mathrm{BDFH} 0}
\end{array}\right],
$$

where $f^{1}(), f^{2}()$, and $f^{3}()$ are the transformation functions which convert the output of bridge (AD value) to force/torque (unit: $\mathrm{KN} / \mathrm{KN} \cdot \mathrm{m}$ ). These functions can be obtained by static calibration. $v F_{\mathrm{CG}}, v F_{\mathrm{AE}}$, and $v F_{\mathrm{BDFH}}$ are the output of $B_{\mathrm{CG}}$ bridge, $B_{\mathrm{AE}}$ bridge, and $B_{\mathrm{BDFH}}$ bridge, respectively, and $F_{\mathrm{CG} 0}$, $F_{\mathrm{AEO}}$, and $F_{\mathrm{BDFH} 0}$ are the initial value of $B_{\mathrm{CG}}$ bridge, $B_{\mathrm{AE}}$ bridge, and $B_{\mathrm{BDFH}}$ bridge in $\mathrm{KN}$, respectively.

It is noticed that $\theta$ reflects the relative angle between $O_{c} X_{c} Y_{c} Z_{c}$ and $O_{w} X_{w} Y_{w} Z_{w}$, so it cannot be measured directly. In order to solve the problem, an absolute encoder is adopted to measure the rotation angle of $O_{c} X_{c} Y_{c} Z_{c}$ and its output is denoted as $\alpha$. When $O_{c} X_{c} Y_{c} Z_{c}$ coincides with $O_{w} X_{w} Y_{w} Z_{w}$, the encoder's output is $\alpha_{0}$. Then (1) can be converted to (2) as shown below:

$$
\begin{aligned}
{\left[\begin{array}{c}
F_{x} \\
F_{z} \\
M_{y}
\end{array}\right]=} & {\left[\begin{array}{ccc}
\cos \left(\alpha-\alpha_{0}\right) & \sin \left(\alpha-\alpha_{0}\right) & 0 \\
-\sin \left(\alpha-\alpha_{0}\right) & \cos \left(\alpha-\alpha_{0}\right) & 0 \\
0 & 0 & 1
\end{array}\right] } \\
& \times\left[\begin{array}{c}
f^{1}\left(v F_{\mathrm{CG}}\right)-F_{\mathrm{CG} 0} \\
f^{2}\left(v F_{\mathrm{AE}}\right)-F_{\mathrm{AE} 0} \\
f^{3}\left(v F_{\mathrm{BDFH}}\right)-F_{\mathrm{BDFH} 0}
\end{array}\right] .
\end{aligned}
$$

In (2), there are four initial values to be solved which are $\alpha_{0}, F_{\mathrm{CG} 0}, F_{\mathrm{AE} 0}$, and $F_{\mathrm{BDFH} 0}$. As shown in Section 1, these initial values change after installation. Therefore, they cannot be 


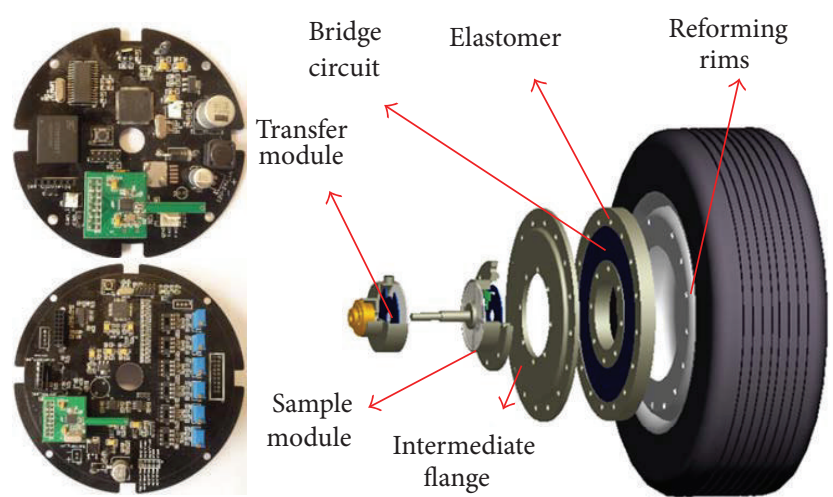

(a) The overall structure of the WFT

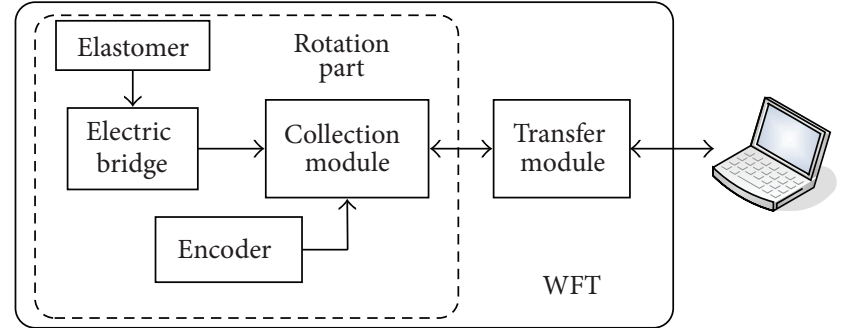

(b) The electrical flowchart

FIGURE 5: Diagram of the adopted 3-axis WFT's structure.
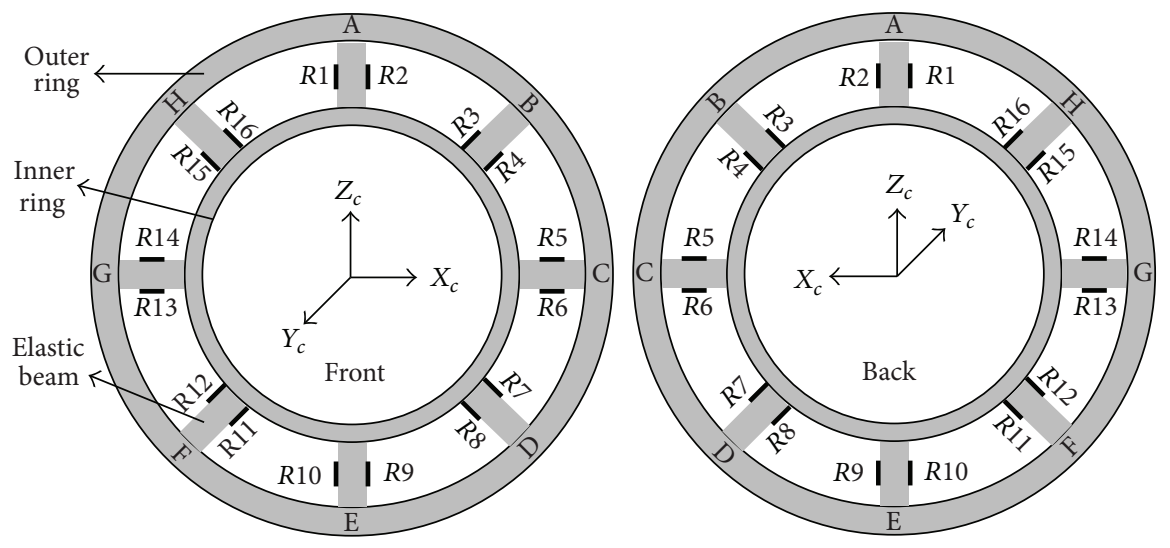

Figure 6: The diagram of the WFT elastic body.

obtained by traditional static calibration method. Consider the following.

(1) For $O_{w} X_{w} Y_{w} Z_{w}$ is a coordinate in concept, it is hard to judge whether $O_{c} X_{c} Y_{c} Z_{c}$ coincides with $O_{w} X_{w} Y_{w} Z_{w}$. Aiming manually is an intuitive approach; however, the elastic body is covered by the intermediate flange as shown in the Figure 5, so aiming manually will cause inevitable error.

(2) When the WFT is installed in the wheel, the output of $B_{\mathrm{BDFH}}$ bridge can be considered as initial value $F_{\mathrm{BDFH} 0}$ if the vehicle is static.

(3) The method shown in (2) is not suitable for $F_{\mathrm{CG} 0}$ and $F_{\mathrm{AEO}}$. When vehicle is static, the component force of vehicle weight is applied to the $B_{\mathrm{CG}}$ bridge and $B_{\mathrm{AE}}$ bridge, then their output depends on vehicle weight and angle $\theta$. Therefore, measuring initial value $F_{\mathrm{CG} 0}$ and $F_{\mathrm{AE} 0}$ correctly is difficult too.

From the description above, it is clear that initial values $\alpha_{0}, F_{\mathrm{CG} 0}$, and $F_{\mathrm{AE} 0}$ cannot be measured directly. Unfaithful initial values will result in the inaccurate longitudinal force $F_{x}$ and vertical force $F_{z}$; therefore how to solve the initial value calibration problem to acquire $\alpha_{0}, F_{\mathrm{CG} 0}$, and $F_{\mathrm{AE} 0}$ with high accuracy is an important work.
According to (2), (3) is obtained as

$$
\begin{aligned}
F_{x}= & \cos \left(\alpha-\alpha_{0}\right)\left(f^{1}\left(v F_{\mathrm{CG}}\right)-F_{\mathrm{CG} 0}\right) \\
& +\sin \left(\alpha-\alpha_{0}\right)\left(f^{2}\left(v F_{\mathrm{AE}}\right)-F_{\mathrm{AE} 0}\right), \\
F_{z}= & -\sin \left(\alpha-\alpha_{0}\right)\left(f^{2}\left(v F_{\mathrm{CG}}\right)-F_{\mathrm{CG} 0}\right) \\
& +\cos \left(\alpha-\alpha_{0}\right)\left(f^{1}\left(v F_{\mathrm{AE}}\right)-F_{\mathrm{AE} 0}\right) .
\end{aligned}
$$

In (3), $\alpha, F_{\mathrm{CG}}, F_{\mathrm{AE}}, f^{1}()$, and $f^{2}()$ can be acquired from the WFT's output and static calibration, and $w=$ $\left(\alpha_{0}, F_{\mathrm{CG} 0}, F_{\mathrm{AE} 0}\right)$ are the variables to be solved. Because the value of $F_{x}$ and $F_{z}$ is unknown, it is difficult to solve the transcendental equations. Intuitively, if the unknowns $F_{x}$ and $F_{z}$ can be removed from (3) by some ways, it is possible to solve the transcendental equations and acquire the initial values. This idea is the basis of the proposed method.

Assuming there is a scene where the longitudinal force applied to the wheel remains unchanged and $\alpha_{k i}, F_{\mathrm{CG} k i}$, and 


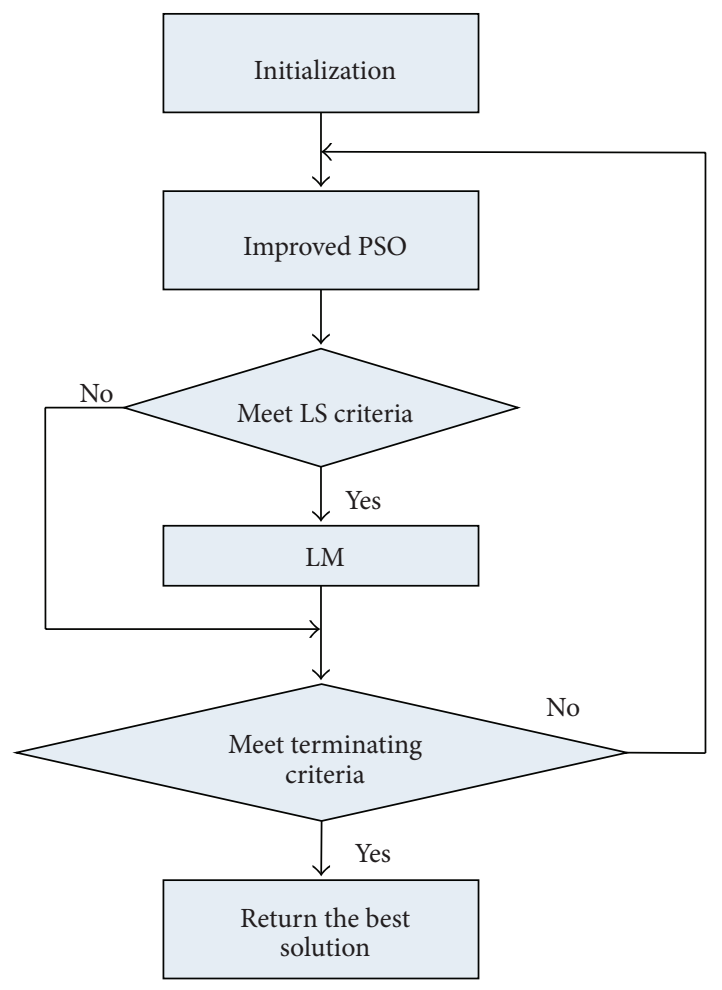

FIgURE 7: The flowchart of the proposed IMAPL.

$F_{\mathrm{AEk} i}$ are the output of the WFT. Then the following equation can be obtained:

$$
\begin{aligned}
F_{x i}-F_{x j}= & -\sin \left(\alpha_{i}-\alpha_{0}\right)\left(f^{1}\left(v F_{\mathrm{CG} i}\right)-F_{\mathrm{CG} 0}\right) \\
& +\cos \left(\alpha_{i}-\alpha_{0}\right)\left(f^{2}\left(v F_{\mathrm{AE} i}\right)-F_{\mathrm{AE} 0}\right) \\
& +\sin \left(\alpha_{j}-\alpha_{0}\right)\left(f^{1}\left(v F_{\mathrm{CG} j}\right)-F_{\mathrm{CG} 0}\right) \\
& -\cos \left(\alpha_{j}-\alpha_{0}\right)\left(f^{2}\left(v F_{\mathrm{AE} j}\right)-F_{\mathrm{AE} 0}\right)=0 .
\end{aligned}
$$

In (4), $i$ is not equal to $j$. For the longitudinal force is constant in the whole process; therefore we can get the following equations:

$$
\begin{gathered}
F_{x 2}-F_{x 1}=0 \\
\vdots \\
F_{x i}-F_{x 1}=0 \\
F_{x i}-F_{x 2}=0 \\
\vdots \\
F_{x i}-F_{x i-1}=0 \\
\vdots \\
F_{x N}-F_{x N-1}=0,
\end{gathered}
$$

where $N$ is the total number of sampled data. Equation (5) is a nonlinear transcendental equation which can be solved by Newton iteration method.

Thus, if we can find a scene where the longitudinal force applied to the wheel is a constant, the unknown variables $w$ can be acquired by solving (5). However, the scene does not exist in reality. Here some similar scenes in real are considered where the longitudinal force applied approximately to the wheel is relatively stable and remains unchanged, for example, the car moves with a constant speed on a flat road. In these real scenes, because of the measurement noise and nonperfect flat road, $\left\{F_{x i}-F_{x j}\right\}$ is not exactly equal to 0 , then the unknown initial variables $w$ cannot be solved correctly by (5). It is clear that with the correct initial variables, the difference between $F_{x i}$ and $F_{x j}$ is small enough. Therefore, to recover the unknown variables $w$ from the sampled data, we convert the problem of solving (5) to the following optimization minimization problem:

$$
w^{*}=\underset{w}{\arg \min }\left(\frac{\left(\sum_{i, j=1}^{N}\left|F_{x i}(w)-F_{x j}(w)\right|\right)}{N}\right) .
$$

As long as the optimization problem is solved, the optimal $w^{*}$ can be acquired and then the initial value $\alpha_{0}, F_{\mathrm{CG} 0}$ and $F_{\mathrm{AE} 0}$ are obtained.

3.2. Optimization by the Memetic Algorithm. Generally, the sampled dataset is large and the noise is inevitably involved. Thus, an efficient and robust optimization method is required to solve the optimization problem, as shown in (6).

In recent years, the Memetic Algorithm (MA) gradually becomes a research hotspot in evolutionary computing and achieves good effect in many applications [17-20]. In the standard flowchart of MA, a local search (LS) strategy is executed after crossover and mutation operations. By using LS strategy to exploit the local fitness landscape, the slow convergence of EA to locate high quality solutions improves. However, the LS strategy takes effect on individuals selected by EA every generation, which means the MA places too much emphasis on exploitation. This operation will change the balance of exploration and exploitation and weaker the fast convergence capability of EA. To solve the problem, an improved MA based on PSO and LM (IMAPL) is presented, which aims to balance exploration and exploitation. The characteristics of IMAPL are

(1) LM algorithm is adopted as the LS strategy;

(2) as one promising EA, an improved PSO algorithm based on immune clone strategy is adopted [21]. The clone and immune operators are integrated into PSO to further improve the exploration capability and diversity;

(3) unlike the classical MA, in IMAPL, LS strategy is isolated from the EA, and LM is adopted to exploit the local fitness landscape only if PSO is considered to have found a promising solution based on LS criteria.

The algorithm flowchart is shown in Figure 7. In the algorithmic framework, there are two criteria. The first one 


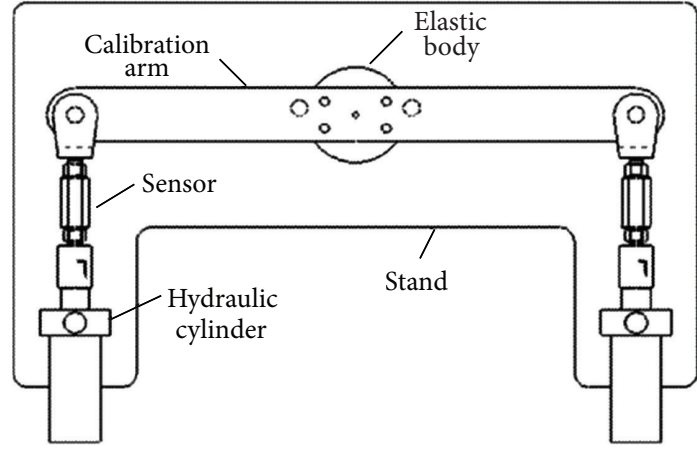

(a)

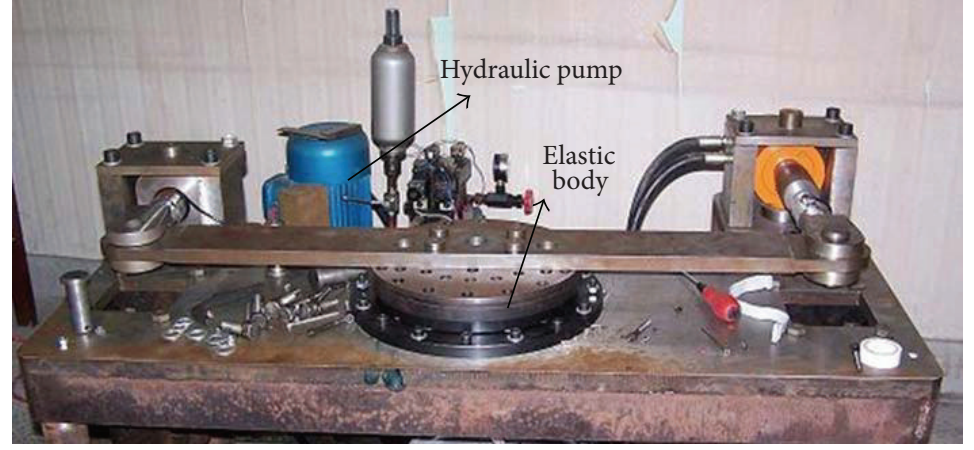

(b)

FIGURE 8: The static calibration platform.
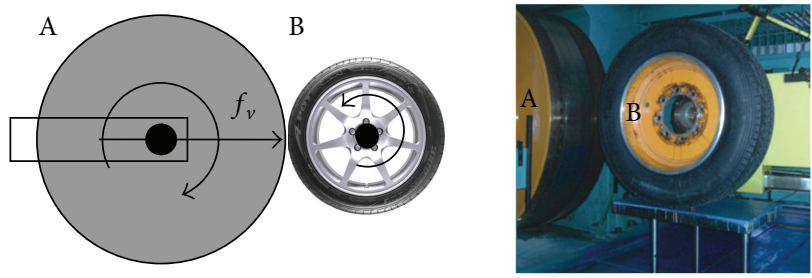

Figure 9: The schematic diagram of VDTS.

is the LS criterion which is used to decide whether or not to exploit LM. The other one is the terminating criterion which is used to decide whether or not to terminate the algorithm. LS criteria is a key part of IMAPL. Let $P_{g}(t)$ and $P_{g}(t)$ denote the global best position of all particles in $t$ th and $(t-1)$ th iterations in the improved PSO algorithm. In LS criteria, if the ratio of the absolute distance between $P_{g}(t-1)$ and $P_{g}(t)$ to $P_{g}(t-1)$ is less than a predetermined threshold $T_{g}$, then it is not wise to apply LM to improve $P_{g}(t)$ since it may lead to the same local optimum. Terminating criteria is simple, it is that when the iteration number reaches a predetermined number $T_{c}$ or the fitness function is less than a predetermined threshold $T_{e}$.

\section{Experiments}

In the experiments, one type of WFT developed by Southeast University is adopted. It is designed for Chevrolet Sail vehicle, and its measuring range is from $0 \mathrm{KN}$ to $14 \mathrm{KN}$. Before the experiment, a static calibration is carried out to acquire the transformation function $f^{1}(), f^{2}()$, and $f^{3}()$, which is shown in (1). As shown in Figure 8, a static calibration platform based on hydraulics is adopted.

Because it is difficult to verify the effect of the proposed initial value calibration method in real vehicle test, a road wheel test-bed with force feedback is adopted. In this paper, the adopted road wheel test-bed is produced by MTS in 1986 and it is called Vehicle Dynamics Test System (abbreviated as VDTS). For security reasons, the physical map of VDTS cannot be public. Figure 9 shows the schematic diagram and the similar equipment. As shown in Figure 9, the main part
TABLE 1: Three groups of force and speed in initial value calculation.

\begin{tabular}{lccc}
\hline & Test 1 & Test 2 & Test 3 \\
\hline Applied force $(\mathrm{kN})$ & 13 & 8 & 4 \\
Rotating speed $(\mathrm{rpm})$ & 20 & 30 & 40 \\
\hline
\end{tabular}

of VDTS is composed of driving wheel A and driven wheel B. The wheel A can drive the wheel B to rotate and apply a force $f_{v}$ to the wheel $\mathrm{B}$ along a horizontal direction simultaneously. The WFT is bolted on the wheel B. For the WFT, the force $f_{v}$ applied to the wheel A can be considered as the longitudinal force $F_{x}$. It is good for us that the force $f_{v}$ can be feedback and measured by VDTS; therefore it can be used to compare with $F_{x}$ solved by the WFT. It should be noted that the solved $F_{x}$ is not exactly equal to the force $f_{v}$. It is because the WFT and the feedback sensor are mounted in different wheels, and $f_{v}$ is not exactly along the $X$-axis of $O_{w} X_{w} Y_{w} Z_{w}$. Nevertheless, by the error between $f_{v}$ and $F_{x}$ solved by the WFT, the performance of the presented method can be evaluated.

4.1. Initial Value Calibration by Means of VDTS. By means of VDTS, it is easy to set up the scenes where the longitudinal force $F_{x}$ applied to the wheel can be regarded as a constant. Firstly, a constant force $f_{v}$ is applied to the wheel B by the wheel A. Secondly the wheel A rotates with constant speed for several seconds, and the data is sampled. The two steps repeat for several times with different force and different speed. It is noticed that because the significant vibration is generated when VDTS runs with high speed, the feedback force will also vibrate obviously. Therefore, the low speed is selected here. In the experiments, three different force and speed are adopted, as shown in Table 1.

From the three groups of sampled data, some good and stable data are selected to construct three datasets separately. The three datasets are substituted in (7) which is derived from (6) to calculate the initial value. Consider

$$
w^{*}=\underset{w}{\arg \min }\left(\frac{\left(\sum_{k=1}^{3} \sum_{i, j=1}^{N_{k}}\left|F_{x i}(w)-F_{x j}(w)\right|\right)}{\sum_{k=1}^{3} N_{k}}\right),
$$

where $N_{k}(k=1,2,3)$ is the number of each dataset. 


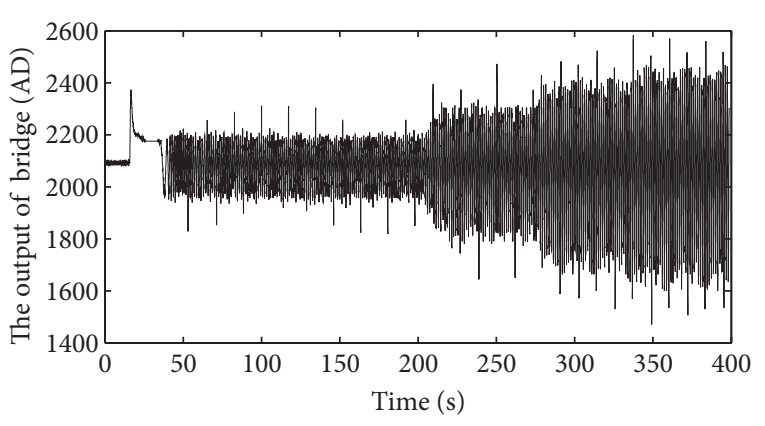

(a) The original outputs of $B_{\mathrm{CG}}$ bridge

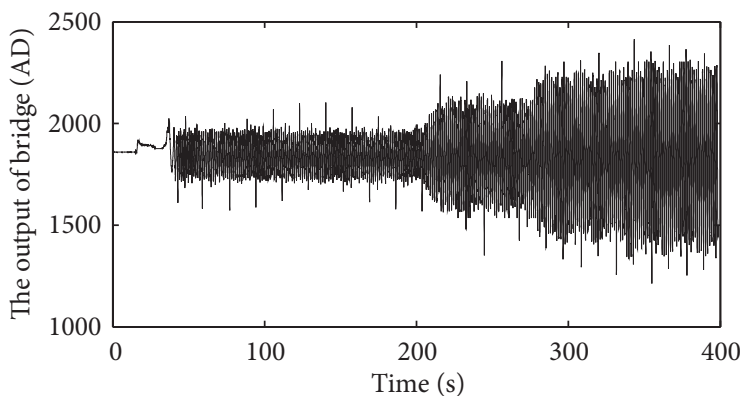

(b) The original outputs of $B_{\mathrm{AE}}$ bridge

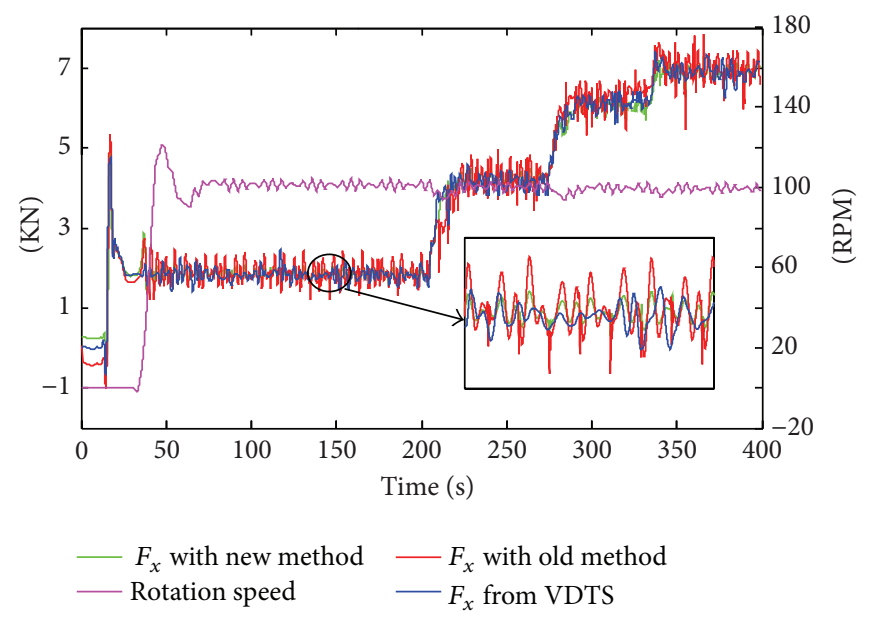

(c) The $F_{x}$ curves with different initial value calibration method

FIgURE 10: The curve of the dynamic test 1.

To solve (7), IMAPL described in Section 3.2 is exploited, and some thresholds are defined in advance. Set $T_{g}=10 \%, T_{c}$ $=200$, and $T_{e}=2 \% \times 14 \mathrm{KN}(14 \mathrm{KN}$ is the maximum measuring range of the adopted WFT). After the optimization, the initial value is acquired as follows, $\alpha_{0}=238.4^{\circ}, F_{\mathrm{CG} 0}=242 \mathrm{~N}$, and $F_{\mathrm{AE} 0}=203 \mathrm{~N}$.

For comparison, the traditional methods are used. Because the dynamic test environment is different from real vehicle, some methods to obtain initial value described in Section 2 cannot be used in VDTS. Here the initial values obtained by traditional methods are denoted as $\left\{\alpha_{0}^{\prime}, F_{\mathrm{CG} 0}^{\prime}, F_{\mathrm{AE} 0}^{\prime}\right\}$. In this experiment, the output of $B_{\mathrm{CG}}$ bridge and $B_{\mathrm{AE}}$ bridge when the WFT is not mounted in the wheel is taken as $F_{\mathrm{CG} 0}^{\prime}$ and $F_{\mathrm{AE} 0}^{\prime}$. This operation is a common method to obtain the initial value for other type sensors. For initial value $\alpha_{0}^{\prime}$, the method proposed by Weigong Zhang is used. To simulate the scene proposed in this method, the wheel B is driven to rotate manually instead of being driven by the wheel A. The initial values $\left\{\alpha_{0}^{\prime}, F_{\mathrm{CG} 0}^{\prime}, F_{\mathrm{AE} 0}^{\prime}\right\}$ acquired are $232.6^{\circ}, 113 \mathrm{~N}$, and $102 \mathrm{~N}$.

It is noticed that the error between $\alpha_{0}$ and $\alpha_{0}^{\prime}$ is relative small. This is because that when the wheel $\mathrm{B}$ is driven to rotate manually, the external force applied to the WFT is small enough which meets the principle of Zhang's method. Meanwhile, the error between $F_{\mathrm{CG} 0}$ and $F_{\mathrm{CG} 0}^{\prime}, F_{\mathrm{AE} 0}$ and $F_{\mathrm{AE} 0}^{\prime}$ is large.
4.2. Dynamic Test by Means of VDTS. In dynamic test, different force and speed are selected which is as follows.

(1) Dynamic test 1 . The force $f_{v}$ applied to the wheel $B$ is set to about $1.8 \mathrm{KN}, 4.1 \mathrm{KN}, 6.1 \mathrm{KN}$, and $6.9 \mathrm{KN}$, successively, with the constant rotating speed which is about 102 RPM.

(2) Dynamic test 2. The force $f_{v}$ applied to the wheel B is set to about $2 \mathrm{KN}, 4 \mathrm{KN}, 5.9 \mathrm{KN}, 8 \mathrm{KN}, 4.8 \mathrm{KN}, 2.2 \mathrm{KN}$, and $0.4 \mathrm{KN}$, successively, with the constant rotating speed which is about 118 RPM.

(3) Dynamic test 3. The force $f_{v}$ applied to the wheel $B$ is set to about $2.3 \mathrm{KN}, 4.2 \mathrm{KN}, 6.1 \mathrm{KN}, 7.3 \mathrm{KN}$, and $5.4 \mathrm{KN}$, successively, with two different rotating speeds which are about 72 RPM and 142 RPM.

The original outputs of $B_{\mathrm{CG}}$ bridge in three dynamic tests are shown in Figures 10(a), 11(a), and 12(a) separately, and the original outputs of $B_{\mathrm{AE}}$ bridge are shown in Figures 10(b), 11(b), and 12(b). The force $f_{v}$, the solved $F_{x}$ by different initial value calibration method, and the rotation speed in three dynamic tests are shown in Figures 10(c), 11(c), and 12(c), respectively. For evaluating the performance of the proposed initial value calibration method, the dynamic errors (absolute maximum error and average error) are exploited. 


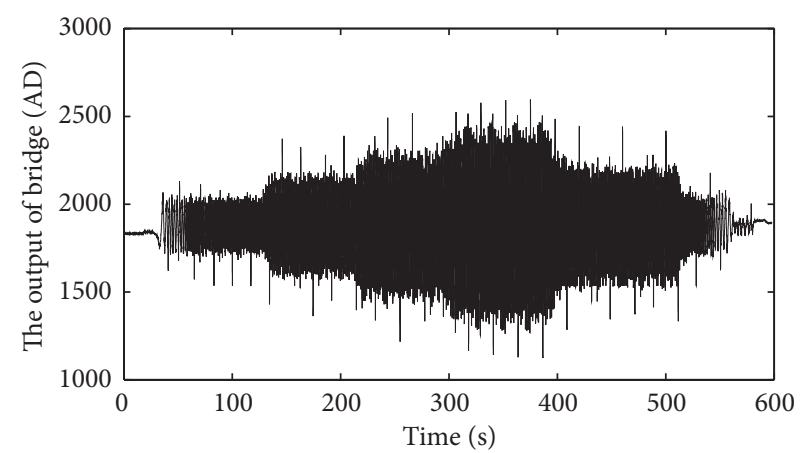

(a) The original outputs of $B_{\mathrm{CG}}$ bridge

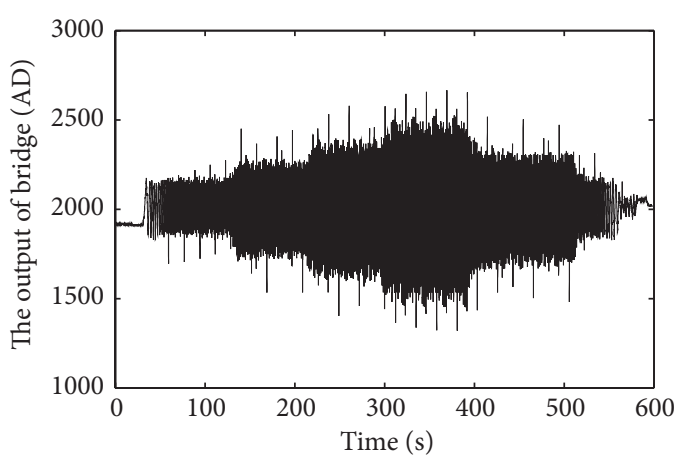

(b) The original outputs of $B_{\mathrm{AE}}$ bridge

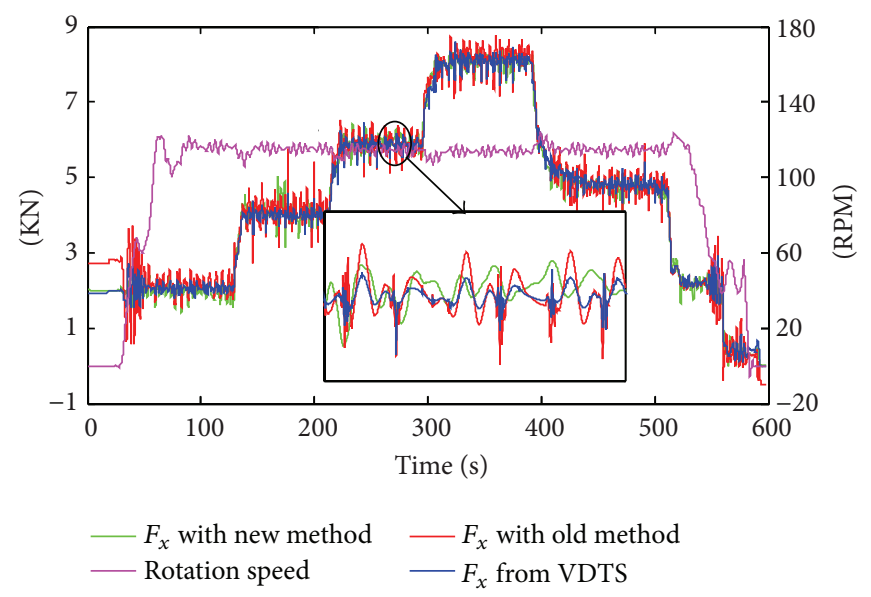

(c) The $F_{x}$ curves with different initial value calibration method

FIgURE 11: The curve of the dynamic test 2.

TABLE 2: The comparison of measuring accuracy based on absolute maximum error (unit: $\mathrm{KN}$ ).

\begin{tabular}{lccc}
\hline & $\begin{array}{c}\text { Dynamic } \\
\text { test 1 }\end{array}$ & $\begin{array}{c}\text { Dynamic } \\
\text { test 2 }\end{array}$ & $\begin{array}{c}\text { Dynamic } \\
\text { test 3 }\end{array}$ \\
\hline The proposed method & 1.03 & 1.13 & 0.87 \\
The traditional method & 1.18 & 1.38 & 1.29 \\
\hline
\end{tabular}

TABLE 3: The comparison of measuring accuracy based on average error (unit: KN).

\begin{tabular}{lccc}
\hline & $\begin{array}{c}\text { Dynamic } \\
\text { test 1 }\end{array}$ & $\begin{array}{c}\text { Dynamic } \\
\text { test 2 }\end{array}$ & $\begin{array}{c}\text { Dynamic } \\
\text { test 3 }\end{array}$ \\
\hline The proposed method & 0.13 & 0.12 & 0.15 \\
The traditional method & 0.31 & 0.27 & 0.42 \\
\hline
\end{tabular}

The dynamic errors of the two initial value calibration methods in three dynamic tests are shown in Tables 2 and 3.

From these figures, it can be seen that the longitudinal force $F_{x}$ with two different initial value method are all closed to the applied force $f_{v}$. Compared with the traditional method in these dynamic tests, it is obvious that the proposed method brings the better solutions than the traditional method.
The absolute maximum errors of the proposed method and that of the old method are all between 10\% and 15\%, and the ratio of the former to the latter is about $70 \% \sim 80 \%$ which means the absolute maximum errors caused by the two methods are close. From Figure 10 to Figure 12 it can be seen that the maximum error mainly happened at the moment when the wheel A just touches and detaches the wheel B or the time that the wheel begins to rotate or stop. At this moment, the vibration is generated which causes the large change of the output of the WFT regardless of which method is adopted. At the same time, the average errors of the proposed method and that of the old method decrease a lot which are less than 3\%, and the ratio of the former to the latter is less than $40 \%$, which means the new method comes with higher accuracy than the previous method with the average errors.

From the analysis of the dynamic test, it is obvious that the proposed initial value calibration method can acquire more accuracy initial value and achieve better performance than the old method.

\section{Conclusions}

In order to increase the measuring accuracy of the WFT, a new dynamic and online method to resolve the initial values of the WFT is presented in this paper. Without any additional 


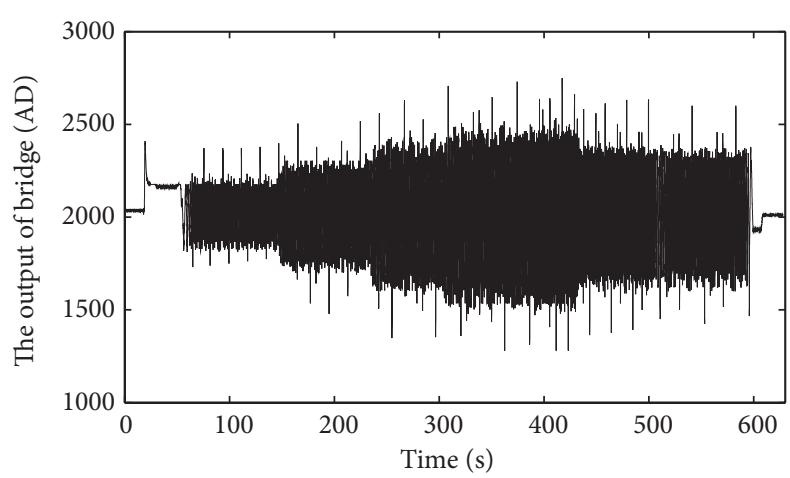

(a) The original outputs of $B_{\mathrm{CG}}$ bridge

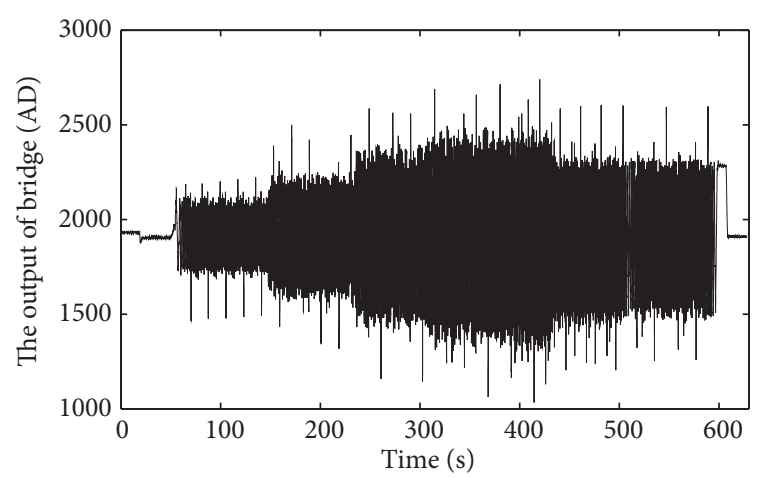

(b) The original outputs of $B_{\mathrm{AE}}$ bridge

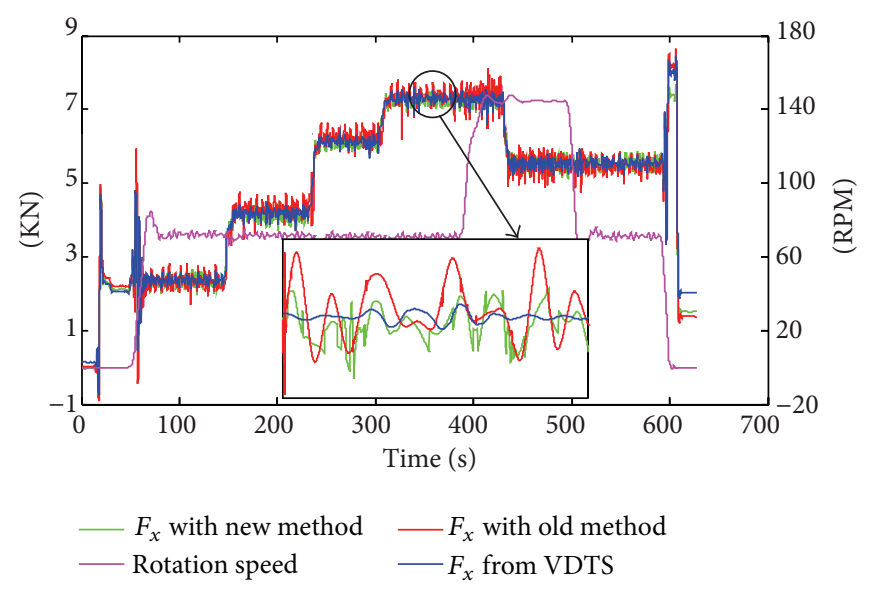

(c) The $F_{x}$ curves with different initial value calibration method

FIGURE 12: The curve of the dynamic test 3.

calibration equipment or manual operation, this method only requires the vehicle mounted with the WFT to be driven on a flat road with constant speed. The theoretical derivation and operation method are introduced. The contrast experiments on the wheel test-bed illustrate its advantage compared to the old ways. With the new method, the initial values are obtained with more accuracy and more convenience, and the measurement accuracy of the WFT is explicitly improved.

\section{Conflict of Interests}

The authors declare no conflict of interests.

\section{Acknowledgment}

This work is supported by National Natural Science Foundation of China (Grant no. 51305078).

\section{References}

[1] M. Corno, M. Gerard, M. Verhaegen, and E. Holweg, "Hybrid ABS control using force measurement," IEEE Transactions on Control Systems Technology, vol. 20, no. 5, pp. 1223-11235, 2012.

[2] X. Zhang, N. Feng, and W. Zhang, "Experimental research on the abs performance based on the wheel forces measured by roadway test," China Mechanical Engineering, vol. 19, no. 6, pp. 751-755, 2008.

[3] W. Cho, J. Yoon, S. Yim, B. Koo, and K. Yi, "Estimation of tire forces for application to vehicle stability control," IEEE Transactions on Vehicular Technology, vol. 59, no. 2, pp. 638-649, 2010.

[4] Y. Wang, W. Zhang, and H. Wang, "Application of wavelet method with nonlinear threshold control in vehicle wheel force signal denoising," in Proceedings of the 4th International Conference on Intelligent Computation Technology and Automation (ICICTA '11), pp. 957-960, Shenzhen, China, March 2011.

[5] G. F. Liu, W. G. Zhang, and Z. G. Li, "Research on static decoupling for multi dimensional wheel force transducer," Instrument Technique and Sensor, vol. 7, pp. 15-18, 2006.

[6] Y. Zhou, W. Zhang, G. Liu, and Z. Li, "Research and development on the vehicle roadway test system based on a new six-component wheel force transducer," China Mechanical Engineering, vol. 18, no. 20, pp. 2510-2514, 2007.

[7] K. Guo, W. Chen, Y. Ji, and J. Li, "Study and design of static calibration system for 6-D force sensor based on virtual instrument technology," in Proceedings of the 10th International Conference on Electronic Measurement and Instruments (ICEMI '11), pp. 81-84, Chengdu, China, August 2011.

[8] H.-M. Zheng, Z.-S. Liu, and Y. Wang, "Study on the method of dynamic characteristic calibration of the 6-aixs wrist force sensor for robot," Acta Metrologica Sinica, vol. 26, no. 1, pp. 4385, 2005. 
[9] R. Sargeant, L. D. Seneviratne, and K. Althoefer, "A 2-axis optical force-torque fingertip sensor for dexterous grasping using linear polarizers," IEEE Transactions on Instrumentation and Measurement, vol. 61, no. 12, pp. 3363-3377, 2012.

[10] J. C. Liu, Y. G. Li, L. Xie, and J. Liu, "Research on static performance calibration system for multi-axis force sensor based on virtual instrument," Transducer and Micro System Technologies, vol. 29, no. 11, pp. 86-89, 2011.

[11] W. G. Zhang, "Study on multi-component wheel force measurement technology," Journal of Jiangsu University, vol. 25, no. 1, pp. 25-28, 2004.

[12] G. F. Liu, S. G. Li, and W. G. Zhang, "Design of acquisition system of weighing calibration equipment for wheel force transducer," Measurement \& Control Technology, vol. 26, no. 10, pp. 18-20, 2007.

[13] J. Wang, L. Jiang, and H. Liu, "Auto static calibration of multiaxis force sensor based on triaxial accelerometer," Chinese Journal of Scientific Instrument, vol. 29, no. 2, pp. 432-435, 2008.

[14] K. Parsa and F. Aghili, "Adaptive observer for the calibration of the force-moment sensor of a space robot," in Proceedings of the IEEE International Conference on Robotics and Automation (ICRA '06), pp. 1667-1673, Orlando, Fla, USA, May 2006.

[15] K. Kim, Y. Sun, R. M. Voyles, and B. J. Nelson, "Calibration of multi-axis MEMS force sensors using the shape-from-motion method," IEEE Sensors Journal, vol. 7, no. 3, pp. 344-351, 2007.

[16] Y. Sun, K. Kim, R. M. Voyles, and B. J. Nelson, "Calibration of multi-axis MEMS force sensors using the shape from motion method," in Proceedings of the IEEE International Conference on Robotics and Automation (ICRA '06), pp. 269-274, Orlando, Fla, USA, May 2006.

[17] Y. S. Ong and A. J. Keane, "Meta-Lamarckian learning in memetic algorithms," IEEE Transactions on Evolutionary Computation, vol. 8, no. 2, pp. 99-110, 2004.

[18] F. Neri and C. Cotta, "Memetic algorithms and memetic computing optimization: a literature review," Swarm and Evolutionary Computation, vol. 2, pp. 1-14, 2012.

[19] Y. K. Richard, R. L. Fung, and Z. B. Jiang, "A memetic algorithm for the open capacitated arc routing problem," Transportation Research Part E, vol. 50, pp. 53-67, 2013.

[20] S. A. Ludwig, "Memetic algorithms applied to the optimization of workflow compositions," Swarm and Evolutionary Computation, vol. 10, pp. 31-40, 2013.

[21] G. Lin, X. Chen, and W. Zhang, "Robust lane detection algorithm based on multiple information fusion and optimizations," Journal of Southeast University (Natural Science Edition), vol. 40, no. 4, pp. 771-777, 2010. 


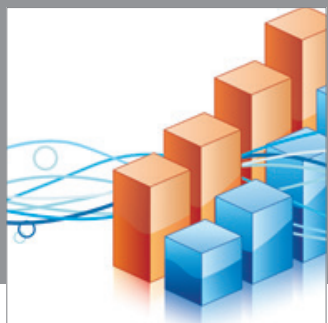

Advances in

Operations Research

mansans

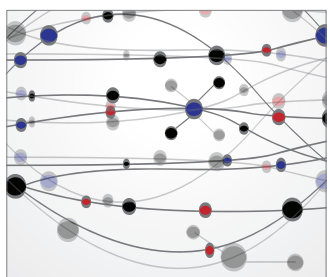

The Scientific World Journal
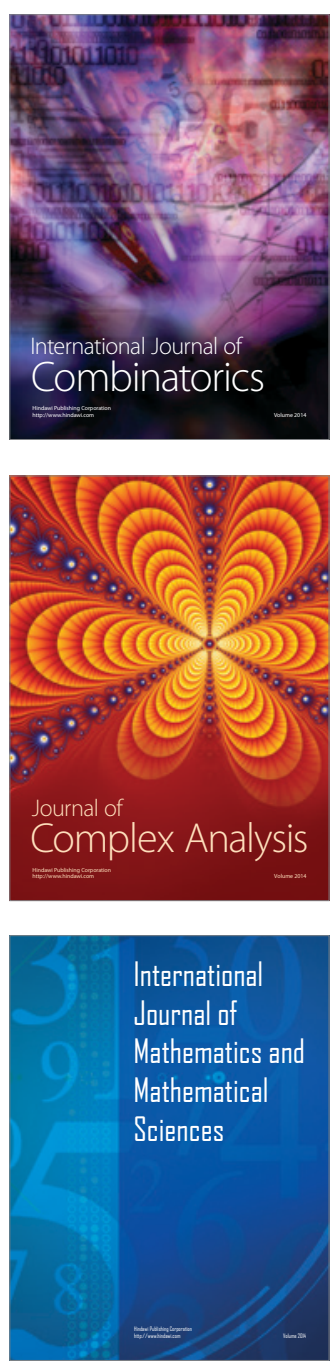
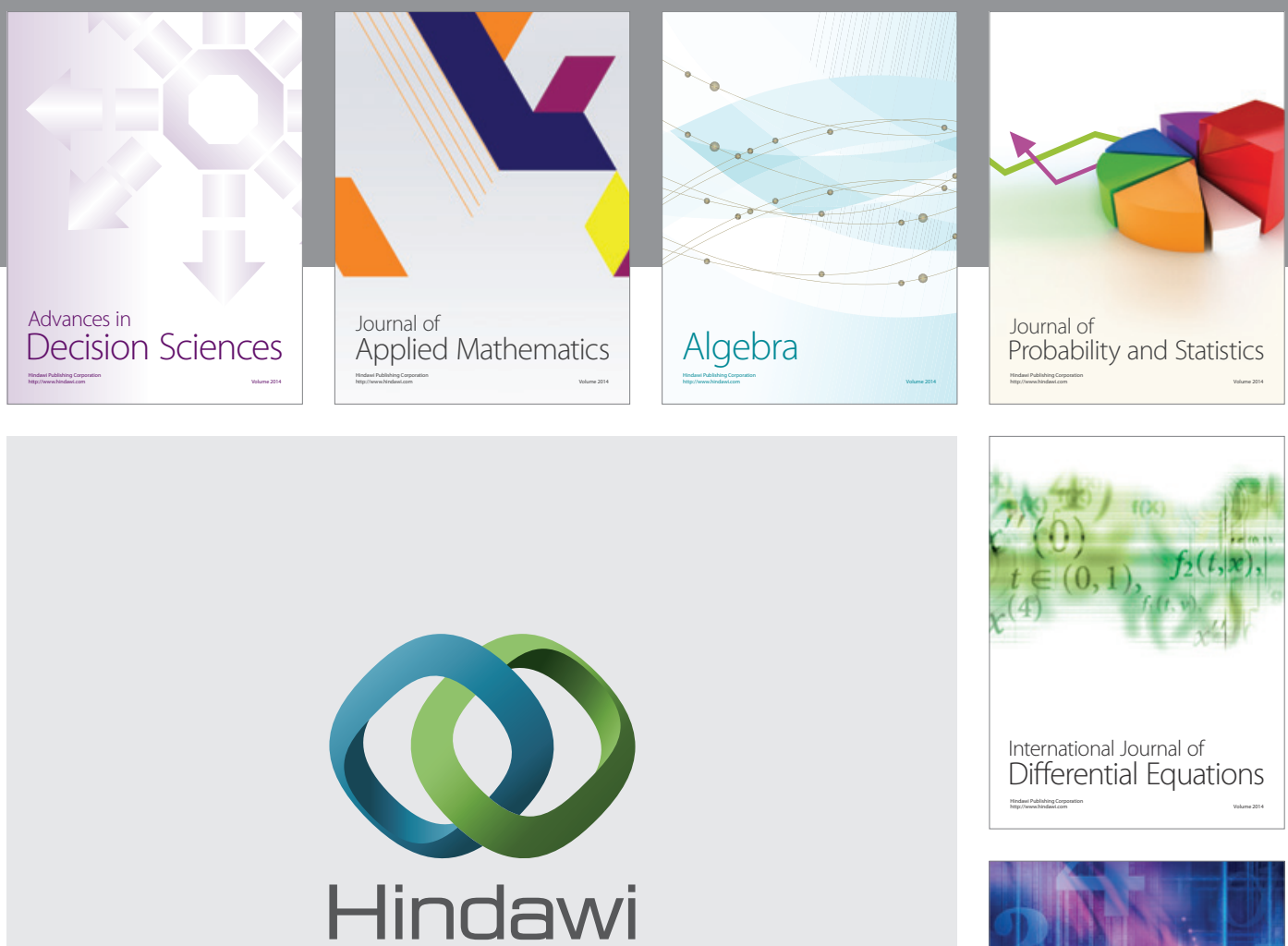

Submit your manuscripts at http://www.hindawi.com
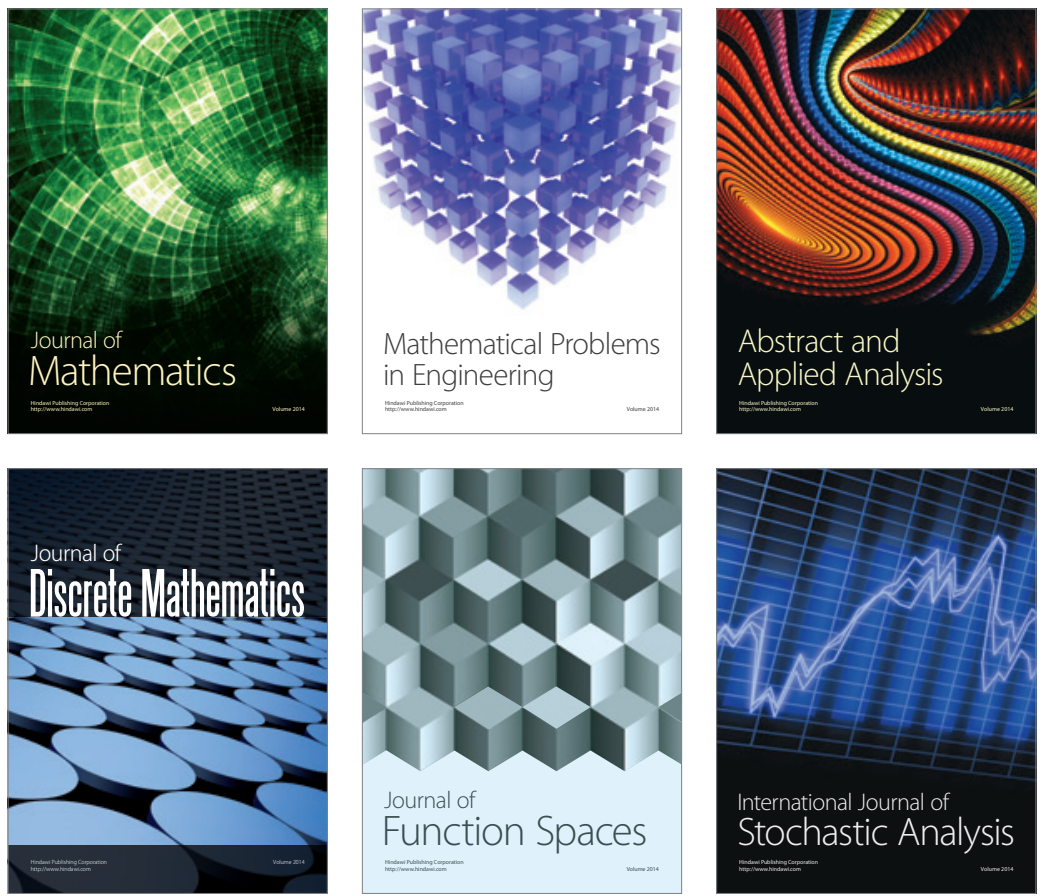

Journal of

Function Spaces

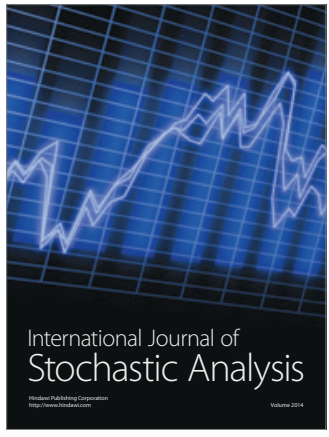

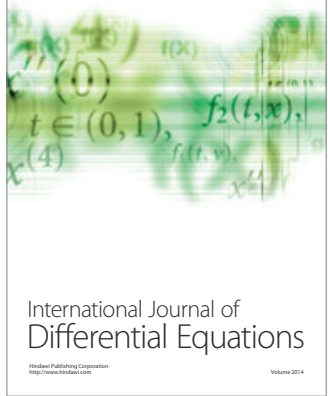
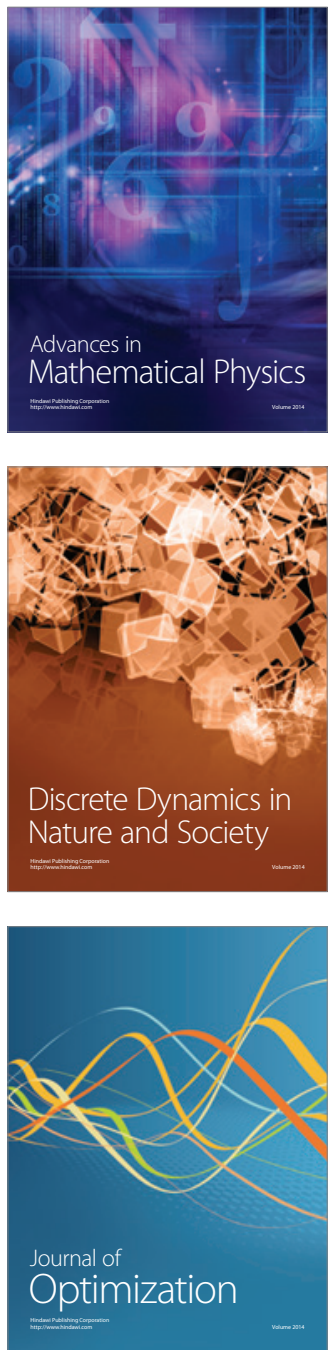